\title{
Could $\mathrm{SiC} \mathrm{A}+\mathrm{B}$ grains have originated in a post-AGB thermal pulse?
}

\author{
Falk Herwig \\ University of Victoria, Victoria, BC, Canada
}

Sachiko Amari

Washington University, St. Louis, USA

Maria Lugaro

IoA, University of Cambridge, Cambridge, UK

Ernst Zinner

Washington University, St. Louis, USA

\begin{abstract}
The carbon and nitrogen isotopic ratios of pre-solar $\mathrm{SiC}$ grains of type $\mathrm{A}+\mathrm{B}$ suggest a proton-limited nucleosynthetic process as encountered, for instance, during the very late thermal pulse of post-AGB stars. We study the nuclear processes during this phase and find carbon and nitrogen isotopic ratios which can reproduce those of $A+B$ grains. These results are still preliminary because they depend on uncertain factors such as the details of mixing during the post-AGB thermal pulse, the rates of some nuclear reactions, and the assumptions on mixing during the progenitor AGB phase.
\end{abstract}

In recent years isotopic analysis of individual pre-solar $\mathrm{SiC}$ grains found in primitive meteorites have established various sub-groups and linked these to different stellar production sites (Zinner, 1998). However, the origin of $\mathrm{SiC} \mathrm{A}+\mathrm{B}$ grains remains elusive. These grains are mainly characterized by low ${ }^{12} \mathrm{C} /{ }^{13} \mathrm{C}$ ratios $(2-10)$ and a wide range of values for the ${ }^{14} \mathrm{~N} /{ }^{15} \mathrm{~N}$ ratio $\left(10^{2}-10^{4}\right)$ (Fig. 1, left panel), while showing close to solar silicon isotopic ratios (Amari et al., 2001). Some stellar sources, such as novae or J-type Asymptotic Giant Branch (AGB) stars, have been considered but cannot explain all the isotopic ratios. These ratios suggest a proton-limited nucleosynthetic process. The conditions for such a process are encountered when small amounts of protons are ingested into a hot carbon-rich region. One stellar evolution phase during which these conditions are met is the very late thermal pulse (VLTP) that occurs in roughly $10 \%$ of all central stars of Planetary Nebulae (Herwig et al., 1999; Herwig, 2001).

We use a fully implicit code to produce one-zone nucleosynthesis models for conditions typical of the VLTP event. The models discussed here are the same as those presented in Herwig, Lugaro \& Werner (this conference). The abundance variations are shown in Fig. 1 (right panel). Due to the large ${ }^{12} \mathrm{C} / \mathrm{H}$ ratio $(\sim 50)$ most protons lead to the production of ${ }^{13} \mathrm{C}$ while few are available 

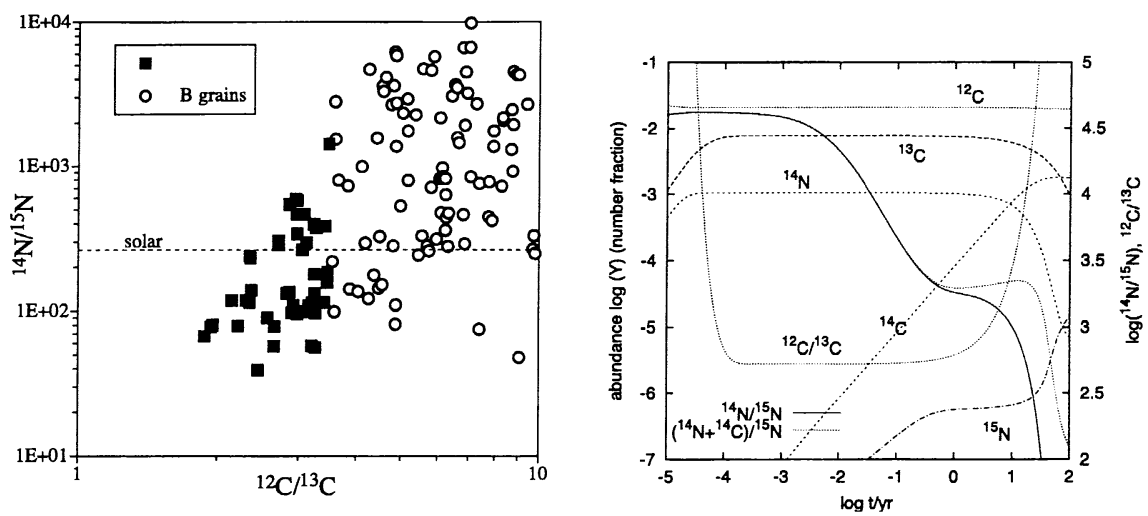

Figure 1. Left: Carbon and nitrogen isotopic ratios of $A+B$ SiC grains. The $\mathrm{C}$ isotopic ratios of some grains are smaller than expected from CNO-cycle equilibrium. Right: Evolution of isotopic abundances according to a one-zone model for typical conditions encountered during the VLTP H-ingestion phase $\left(T_{8}=1.25, X(H)_{\text {initial }}=0.01\right)$.

for the synthesis of a smaller amount of ${ }^{14} \mathrm{~N}$. Neutrons released by the ${ }^{13} \mathrm{C}(\alpha, \mathrm{n})$ reaction are captured predominantly by ${ }^{14} \mathrm{~N}$. Neutron captures by this isotope create ${ }^{14} \mathrm{C}$ via $(\mathrm{n}, \mathrm{p})$ and ${ }^{15} \mathrm{~N}$ through $(\mathrm{n}, \gamma)$. After $t=1 \mathrm{yr}{ }^{15} \mathrm{~N}$ is destroyed by the $(\mathrm{p}, \alpha)$ reaction. The ${ }^{14} \mathrm{~N} /{ }^{15} \mathrm{~N}$ ratio is very sensitive to this and to the ${ }^{14} \mathrm{~N}(\mathrm{n}, \mathrm{p}){ }^{14} \mathrm{C}$ reaction. The final results depend on the chosen temperature as well as on the amount of hydrogen assumed to enter the burning layer. These quantities depend on the mass and metallicity of the parent star and on the details of convective mixing which are still not well known. For example, the $T_{8}=1.35, \mathrm{X}(\mathrm{H})=0.008$ model results after a time of $0.1 \mathrm{yr}$ in ${ }^{12} \mathrm{C} /{ }^{13} \mathrm{C} \sim 2.5$ and ${ }^{14} \mathrm{~N} /{ }^{15} \mathrm{~N} \sim 1000$. Another source of uncertainty is introduced by the choice of the initial Abundances, which depend on the assumptions about mixing during the progenitor AGB phase. Our models can be extended to evolutionary phases of other objects such as accreting white dwarfs, and we plan to include other isotopic ratios in the study, in particular those of silicon.

Acknowledgments. F.H. would like to thank D.A. VandenBerg for support through his Operating Grant from the Natural Science and Engineering Research Council of Canada.

\section{References}

Amari, S., Nittler, L. R., Zinner, E., Lodders, K., \& Lewis, R. S. 2001, ApJ, 559, 463 Herwig, F. 2001, ApJ Lett., 554, L71

Herwig, F., Blöcker, T., Langer, N., \& Driebe, T. 1999, A\&A, 349, L5

Zinner, E. 1998, Ann. Rev. Earth Planet. Sci., 26, 147 